\title{
Apparent absence of Giardia infections among children under 5-years of age with acute watery diarrhoea in Abakaliki, Nigeria
}

\section{Original Paper}

Cite this article: Efunshile AM, Ezeanosike $\mathrm{O}$, Onyekachi ONI, Ugwu MI, König B, Robertson LJ (2019). Apparent absence of Giardia infections among children under 5 -years of age with acute watery diarrhoea in Abakaliki, Nigeria. Epidemiology and Infection 147, e58, 1-5. https://doi.org/10.1017/ S0950268818003151

Received: 20 August 2018

Revised: 5 October 2018

Accepted: 24 October 2018

Key words:

Cryptosporidium; diarrhoea; Giardia duodenalis; parasitic disease epidemiology and control

Author for correspondence:

L. J. Robertson,

E-mail: lucy.robertson@nmbu.no

\author{
A. M. Efunshile ${ }^{1,2}$, O. Ezeanosike ${ }^{3,4}$, O. N. I. Onyekachi' ${ }^{1}$ M. I. Ugwu ${ }^{1}$, B. König ${ }^{5}$ and \\ L. J. Robertson ${ }^{6}$
}

\begin{abstract}
${ }^{1}$ Department of Medical Microbiology, Ebonyi State University, Abakaliki, Nigeria; ${ }^{2}$ Department of Medical Microbiology, Federal Teaching Hospital, Abakaliki, Nigeria; ${ }^{3}$ Department of Paediatrics, Federal Teaching Hospital, Abakaliki, Nigeria; ${ }^{4}$ Department of Paediatrics, Ebonyi State University, Abakaliki, Nigeria;

${ }^{5}$ Institute of Medical Microbiology and Epidemiology of Infectious Diseases, University Teaching Hospital, Leipzig, Germany and ${ }^{6}$ Parasitology, Department of Food Safety and Infection Biology, Faculty of Veterinary Medicine, Norwegian University of Life Sciences, PO Box 369 Sentrum, 0102 Oslo, Norway
\end{abstract}

\begin{abstract}
Although the impact of diarrhoeal disease on paediatric health in Nigeria has decreased in recent years, it remains an important cause of morbidity and mortality in children under 5 years. Rotavirus is recognised as an important aetiological agent, but information on the contribution of intestinal protozoa to watery diarrhoea in this age group in Nigeria is scarce. In this cross-sectional study, faecal samples from children admitted to healthcare centres in Abakaliki, Nigeria with acute watery diarrhoea $(N=199)$ and faecal samples from age-matched controls $(N=37)$ were examined for Cryptosporidium and Giardia using immunofluorescent antibody testing and molecular methods. Cryptosporidium was identified in 13 case samples (6.5\%) and no control samples. For three samples, molecular characterisation indicated C. hominis, GP60 subtypes IaA30R3, IaA14R3 and IdA11. Giardia was not detected in any samples. This contrast in prevalence between the two intestinal protozoa may reflect their variable epidemiologies and probably differing routes of infection. Given that these two parasitic infections are often bracketed together, it is key to realise that they not only have differing clinical spectra but also that the importance of each parasite is not the same in different age groups and/or settings.
\end{abstract}

\section{Introduction}

In Nigeria, deaths from diarrhoea among children below the age of 5 years decreased by just over 20\% between 2005 and 2015, but nevertheless remained substantial at 327.6 per 100000 children; in contrast, the global mortality from diarrhoea in children in this age group is estimated at 74.3 per 100000 [1]. Among the aetiologies associated with mortality due to diarrhoea in Nigeria in children under 5 years, rotaviral enteritis had the highest impact (45\%), with cryptosporidiosis considered responsible for $14.3 \%$ [1]. In the global enteric multicentre study (GEMS) in which the under-5 years age group was stratified into three age groups (under 11 months, 12-23 months, and 24-59 months), it was noticed that at most study sites included, Cryptosporidium tended to be unimportant as a diarrhoeal pathogen in the oldest age stratum, with the greatest burden found in the youngest age groups [2]. An extended analysis has shown that: (1) diarrhoeal diseases in general in children under 5-years has a greater impact regarding long-term health burden than previously estimated [3], and (2) the considerable short-term impact of acute cryptosporidiosis on morbidity in this age group underestimates the true burden by $153 \%$ [4].

Another intestinal protozoan parasite that is often considered together with Cryptosporidium, is Giardia duodenalis. Although infection with Giardia is known to cause diarrhoea, its role in childhood diarrhoea is less certain and it tends not to be associated with increased mortality. One study estimated that in the World Health Organization AFRO region (which includes Nigeria), in the under 5-years age group, around 4-times more cases of diarrhoea were due to Giardia infection than due to Cryptosporidium infection [5]. However, the GEMS study found that there was no significant association between Giardia and symptoms of moderate-to-severe diarrhoea in the cohorts of children under 5-years included in the study and, indeed, in univariate analyses of the oldest age stratum (12-59 months) there was a significantly higher probability of identifying Giardia in controls than in patients in most of the study sites, including those in Africa [2]. A previous meta-analysis of published data had also concluded that although Giardia is associated with persistent diarrhoea in children in developing countries, it does not generally cause acute paediatric diarrhoea among infants and children in those 
countries [6]. A follow-up study of Israeli-Arab children aged 2.34.7 years indicated that infection with Giardia may even lower the risk of acute diarrhoea within this age group [7].

Our study provides further data on the role of both Cryptosporidium and Giardia in paediatric diarrhoea in Nigeria. Furthermore, efforts were made to identify any risk factors for infection with either of these parasites and information regarding symptoms and any home treatment prior to admission was also collected.

\section{Methods}

\section{Study design and setting}

This was a cross-sectional study carried out at the two large healthcare centres within Abakaliki, Nigeria. Abakaliki is the capital city of Ebonyi State, SE Nigeria, approximately $650 \mathrm{~km}$ east of Lagos; in 2006 the population of Abakaliki was around 152000 persons.

Stool samples were collected between the months of December 2016 and March 2017, which coincided with the dry season of the year. Caregivers (parents/guardians) of all the diarrhoeic children fulfilling the inclusion criteria for the study and admitted to the healthcare centres during this period were invited to have their children enrolled in our study. Participation was initiated on receipt of written, informed consent from the caregivers. A Strobe checklist was completed for this study and is included as Supplementary Material, which is available via the Cambridge Core website.

\section{Study population}

Children included in the study were 5-years-old or younger and had been diagnosed with acute watery diarrhoea by the managing paediatricians. Children co-infected with malaria, respiratory tract infections, or other disease conditions were excluded.

\section{Ethical considerations}

Approval for the study was given by the Review and Ethics Committee of the Federal Teaching Hospital, Abakaliki. An informed consent form was signed by parents/guardians prior to enrolment in the study; information in the form made clear that participation in the study was voluntary and participants could opt out of the study at any time without prejudice to the quality of treatment received by their children. Questionnaires designed for data collection were coded to be anonymous such that it was not possible to identify individual patients. Information was collected using the language that the caregivers felt most comfortable using.

\section{Questionnaire}

Data collected by questionnaire include sociodemographic details as well as information regarding the particular episode of diarrhoea.

\section{Sample collection and analysis}

In addition to routine diagnostics conducted at the healthcare centres, stool samples were collected from 200 cases and 37 agematched controls (children attending the routine immunization clinics at the same healthcare centres treating the diarrhoea case- patients, with no diarrhoea and apparently healthy; all control children were 5 -years or younger, with over $50 \%$ younger than 11-months old). Samples were preserved in absolute ethanol and transported to the Parasitology Laboratory of the Norwegian University of Life Sciences. Here, each sample was homogenised, washed twice in laboratory-grade water and concentrated by centrifugation. Detection of Cryptosporidium oocysts and Giardia cysts was conducted by standard immunofluorescent antibody test (IFAT) on $10 \mu \mathrm{l}$ subsamples that were air-dried, methanol-fixed and stained with FITC-labelled monoclonal antibody (Mab: Aqua-glo, Waterborne Inc., New Orleans, USA) and 4',6-diamidino-2-phenylindole (DAPI). Prepared slides were examined by fluorescence microscopy, using the appropriate filters, at magnifications of $200 \times$ and $400 \times$ and Cryptosporidium oocysts and/or Giardia cysts enumerated and DAPI inclusion recorded.

DNA was extracted from all those samples that were positive for Cryptosporidium oocysts and every fourth of the other samples (both cases and controls; a total of 59 cases samples and nine control samples) by resuspending $50 \%$ of the remaining pellet in $100 \mu \mathrm{l}$ Tris-EDTA buffer and heating for $1 \mathrm{~h}$ in a heat block set at $100^{\circ} \mathrm{C}$. DNA was then isolated using a QIAmp DNA minikit (QIAGEN GmbH, Germany) following the manufacturer's protocol.

For those samples that were positive for Cryptosporidium, fragments of two genes were used for molecular investigations; these were the SSU rRNA gene (approximately $800 \mathrm{bp}$ ) and the GP60 gene (approximately $950 \mathrm{bp}$;), using published primers and protocols $[8,9]$. For all samples from which DNA had been extracted, PCR with primers targeting the SSU rRNA gene of Giardia was also conducted using published primers and protocols [10]. Each set of PCR included a negative (laboratory-grade water) control and a positive control.

PCR amplification products from positive samples were purified (High Pure PCR product purification kit, Roche Applied Science) according to the manufacturer's protocol and sequenced on both strands at a commercial facility (Macrogen, South Korea). Chromatograms were examined and sequences adjusted manually. Sequence searches were conducted using BLAST (http://blast.ncbi.nlm.nih.gov/Blast.cgi) and, in addition, for GP60 sequences, the $5^{\prime}$ end was manually checked and tandem repeats of the serine-coding trinucleotides enumerated to determine subtype family.

Analysis of the samples for viral pathogens was conducted at the Institute of Virology, University of Leipzig, Germany using multiplex real-time polymerase chain reaction (PCR) standard protocol; the detailed data about viruses are intended for separate presentation.

\section{Data handling and statistical analysis}

Data were compiled in an excel database; and then exported to SPSS version 24 for analysis. Associations were investigated by contingency table analysis (Fisher's Exact Test).

\section{Results}

\section{Study population, symptoms and case management}

Of the 200 case samples collected, one was not suitable for analysis due to specimen leakage; hence 199 cases and 37 controls were included in our final analysis. 
The majority of the study participants were males $(60.8 \%)$ and within the age range of $0-11$-months-old (68.4\%). Most participants came from rural areas and were not exclusively breastfed (72.0\%), (Table 1).

In most of the children, the diarrhoea was associated with fever (98.7\%) and vomiting (92.5\%), and although mucus in faeces was common, only $5 \%$ passed blood-stained stools (Table 2).

In the children's homes, oral rehydration solutions were commonly used (98.0\%), whereas the use of zinc tablets (98\%), intravenous fluids (92.5\%) and antibiotics (87.0\%) were the commonest hospital management practices (Table 3 ).

\section{Aetiology of diarrhoea and occurrence of Cryptosporidium and Giardia}

We did not detect Giardia cysts by IFAT in any of the stool samples, cases or controls and PCR directed towards the Giardia SSU rRNA gene were also negative for the nine control samples and 59 case samples tested. Thus, the prevalence of Giardia infection in this cohort was considered to be $0.00 \%$ (95\% Confidence Intervals (CI) $0.00-1.89$ ).

We did not detect Cryptosporidium oocysts in any of the control samples, but oocysts were identified in 13 of the 199 stool samples from cases $(6.5 \%$; $95 \%$ CI $3.86-10.85)$. Of these positive samples, five contained low numbers of oocysts (20-30 oocysts in the whole slide), seven had moderate numbers of oocysts (up to five oocysts per field of view) but five of these exhibited poor or absent DAPI staining and one sample had very high numbers of oocysts. The number of oocysts per gram of faecal sample could not be estimated due to lack of information on the quantity of stool sample preserved in ethanol.

Of the 13 Cryptosporidium-positive samples, for all of which molecular characterisation was attempted, sequences were obtained only for three (the sample with high numbers of oocysts and the two samples with moderate numbers of oocysts and good DAPI staining). All three were Cryptosporidium hominis, two were of GP60 subtype family Ia (IaA30R3 and IaA14R3) and one of GP60 subtype Id (IdA11).

No associations between Cryptosporidium infection and other variables (age, gender, rural or urban residence) were identified.

Rotavirus was found in $92.3 \%$ of the cases; other agents detected included Enterovirus (20.8\%), Astrovirus (8.6\%), Parechovirus (8.1\%) and Sapovirus (2.7\%). Ascaris eggs and Entamoeba spp. cysts were also seen in $0.5 \%(1 / 199)$ and $1.0 \%(2 / 199)$ of cases, respectively.

\section{Discussion}

The results reported here support those of earlier studies that suggest that in countries such as Nigeria, Giardia infection is not associated with paediatric diarrhoea. Indeed, a meta-analysis of case-control and cohort studies has indicated that there is a significant inverse association between acute diarrhoea and Giardia in stools in children in non-industrialised settings [6]. Thus, these studies have also often shown that control subjects (without diarrhoea) are more frequently infected with Giardia, suggesting that Giardia may mediate a protective effect (e.g. [7, 11-14]), but this was not seen in our study. The nature of the proposed protective effect suggested from other studies is unclear, although changes in mucosal immunity or suppression of other enteric pathogens have been suggested; it is worth noting that a study from Tanzania found that the apparently protective effect of Giardia infection
Table 1. Descriptive characteristics of cases and potential risk factors for infection

\begin{tabular}{|c|c|}
\hline Parameter & Number (\%) \\
\hline \multicolumn{2}{|l|}{ Age group } \\
\hline $0-11$ months & $136(68.4)$ \\
\hline $12-24$ months & $56(28.1)$ \\
\hline $25-60$ months & $7(3.5)$ \\
\hline \multicolumn{2}{|l|}{ Gender } \\
\hline Male & $121(60.8)$ \\
\hline Female & $78(39.2)$ \\
\hline \multicolumn{2}{|c|}{ Highest level of maternal education } \\
\hline No formal education & $52(26.1)$ \\
\hline Primary School & $9(4.5)$ \\
\hline Secondary school & $108(54.3)$ \\
\hline Tertiary level & $30(15.1)$ \\
\hline \multicolumn{2}{|l|}{ Place of residence } \\
\hline Rural & $111(55.8)$ \\
\hline Urban & $88(44.2)$ \\
\hline \multicolumn{2}{|c|}{ Major source of drinking water } \\
\hline Factory packaged water & $155(77.9)$ \\
\hline Borehole & $37(18.7)$ \\
\hline Rain & $3(1.5)$ \\
\hline Stream & $2(1.0)$ \\
\hline Well & $2(1.0)$ \\
\hline \multicolumn{2}{|l|}{ Type of toilet facility } \\
\hline Water closet & $128(64.3)$ \\
\hline Pit latrine & $33(16.6)$ \\
\hline Bush & $38(19.1)$ \\
\hline \multicolumn{2}{|l|}{ Exclusively breastfed } \\
\hline Yes & $56(28.1)$ \\
\hline No & $144(71.9)$ \\
\hline
\end{tabular}

Table 2. Clinical features associated with the diarrhoeal cases

\begin{tabular}{lc}
\hline Clinical feature & Number (\%) \\
\hline Fever & $196(98.5)$ \\
\hline Vomiting & $184(92.5)$ \\
\hline Mucus in stool & $140(70.4)$ \\
\hline Blood in stool & $10(5.0)$ \\
\hline Diarrhoeal persistence for over 1 week & $52(26.1)$ \\
\hline
\end{tabular}

in children of under 6-years was abrogated when multi-nutrient supplementation was used [15]. An alternative possibility is that in profuse watery diarrhoea caused by any aetiology (bacteriological, viral, parasitological), any resident Giardia trophozoites might be 'washed out' before they have time to encyst, hence the lack of Giardia cysts in the samples of diarrhoeic patients, including in our study. It could then be argued that Giardia 
Table 3. Case management practices used for paediatric acute watery diarrhoea at home and in the hospitals

\begin{tabular}{lc}
\hline Case management practices & Number (\%) \\
\hline At home & $118(59.3)$ \\
\hline Continuous feeding & $31(15.6)$ \\
\hline Non-prescription anti-diarrhoea drugs & $195(98.0)$ \\
\hline Oral rehydration solutions & $184(92.5)$ \\
\hline In hospital & $174(87.4)$ \\
\hline Intravenous fluid & $73(36.7)$ \\
\hline Antibiotics & $194(98.0)$ \\
\hline Probiotics & $163(81.9)$ \\
\hline Zinc tablets
\end{tabular}

DNA could have been detected; however, as the samples were collected from children attending healthcare centres due to diarrhoeal disease, it may be likely that any resident Giardia are washed out in early bouts of diarrhoea prior to admission to the healthcare centre and collection of the faecal sample. Such a situation could also, perhaps, explain why previous studies [7, 11-14] have suggested that Giardia infection may exert a protective effect.

Thus, the apparent lack of any Giardia-positive samples in this cohort (cases and controls) may not be as surprising as first appears. Indeed, giardiasis itself is associated with fatty, rather than watery diarrhoea. Preservation in ethanol has been recommended for faecal samples possibly containing Giardia cysts, for subsequent detection by either microscopy (IFAT) or PCR [16]. Nevertheless, degradation during transport cannot be completely excluded as the reason for the lack of detection of Giardia, either cyst structures detected by IFAT, or DNA that can be amplified by targeted PCR. However, this seems unlikely given the detection of Cryptosporidium in some samples. Indeed, previous studies from Nigeria investigating infections in children under 5 -years of age have tended to detect Giardia cases at a relatively low prevalences (e.g. one Giardia-positive 6-month-old with enteritis among six children below 2 years [17]; one child (below 4-years of age) among 215 children with diarrhoea and no Giardia infections among 100 age-matched controls without diarrhoea [18]). However, investigations amongst older children (school-age children, 7-17 years) have tended to show a higher prevalence of Giardia infection, e.g., 12.3\% [19] and 37.2\% [20]; in neither of these studies from school-age children was diarrhoea reported among the subjects.

The $6.5 \%$ prevalence of Cryptosporidium infection in our study was within the range reported by other studies from Nigeria in children aged below 5 years. For example, a 4.8\% prevalence among 165 children below 5 -years of age with diarrhoea was found in Jos, with C. hominis the predominant species, as we found, and subtype Id [21]; a 5.6\% prevalence of Cryptosporidium was reported in children with diarrhoea below 6-years of age from Ebonyi state, with both C. hominis and C. parvum identified and, among the C. hominis isolates, four different GP60 subtypes, including two in the Ia family [22]; a prevalence of Cryptosporidium infection was found in $11.1 \%$ of 21 diarrhoeic children less than 12 months of age from Oyo state; two of the isolates were C. hominis, both subtype IaA24R3 [23].
Our data support the higher occurrence of $C$. hominis species in Africa, despite just under $50 \%$ of the infected children (6 of 13) living in rural areas, where C. parvum may be more common due to associations with animal infections. However, the data provide no information regarding likely vehicles for infection and, given the low number of isolates characterised using molecular methods, our data should be treated with caution regarding extrapolation to other settings.

In conclusion, our results demonstrate that although viral infections appear to be of greatest importance in paediatric diarrhoea in this setting, Cryptosporidium infection is also of relevance. However, Giardia infection seems to be of limited importance in this age group of children, both for asymptomatic children and those with watery diarrhoea. Given that Giardia infections occur relatively frequently in older children, we speculate that our data probably reflect the differing epidemiologies and potentially differing transmission routes for these two parasites. This is important, as many studies tend to consider both Cryptosporidium and Giardia as being very similar epidemiologically, probably due to the fact that they are both intestinal protozoan parasites (albeit of widely differing phylogeny and biology) and have both been associated with waterborne outbreaks.

Supplementary material. The supplementary material for this article can be found at https://doi.org/10.1017/S0950268818003151.

Author ORCIDs. (D) L. J. Robertson 0000-0001-5186-4421.

Acknowledgements. We would like to thank all the parents, guardians and children who participated in this study for their ready cooperation. We also acknowledge the support of Dr Nwangwu Emeka and the nurses at our study sites. In Leipzig, we are grateful for the support of Dr Arne Rodloff and we note that Dr Pikka Jokelainen at Statens Serum Institut, Copenhagen provided support from Denmark. Dr Kjersti Selstad Utaaker and Dr Kristoffer Relling Tysnes are gratefully acknowledged for some technical support at the Norwegian University of Life Sciences. This research received no specific grant from any funding agency, commercial or not-for-profit sectors.

Conflict of interest. None.

\section{References}

1. GBD Diarrhoeal Diseases Collaborators (2017) Estimates of global, regional, and national morbidity, mortality, and aetiologies of diarrhoeal diseases: a systematic analysis for the Global Burden of Disease Study 2015. The Lancet. Infectious Diseases 17, 909-948.

2. Kotloff KL et al. (2013) Burden and aetiology of diarrhoeal disease in infants and young children in developing countries (the Global Enteric Multicenter Study, GEMS): a prospective, case-control study. The Lancet 382, 209-222.

3. Troeger C et al. (2018) Global disability-adjusted life-year estimates of long-term health burden and undernutrition attributable to diarrhoeal diseases in children younger than 5 years. The Lancet. Globalization and Health 6, e255-e269.

4. Khalil IA et al. (2018) Morbidity, mortality, and long-term consequences associated with diarrhoea from Cryptosporidium infection in children younger than 5 years: a meta-analyses study. The Lancet. Global Health 6, e758-e768.

5. Pires SM et al. (2015) Aetiology-specific estimates of the global and regional incidence and mortality of diarrhoeal diseases commonly transmitted through food. PLoS One 10, e0142927.

6. Muhsen K and Levine MM (2012) A systematic review and meta-analysis of the association between Giardia lamblia and endemic pediatric diarrhea in developing countries. Clinical Infectious Diseases 55(suppl. 4), S271S293. 
7. Muhsen K, Cohen D and Levine MM (2014) Can Giardia lamblia infection lower the risk of acute diarrhea among preschool children? Journal of Tropical Pediatrics 60, 99-103.

8. Xiao L et al. (1999) Phylogenetic analysis of Cryptosporidium parasites based on the small-subunit rRNA gene locus. Applied and Environmental Microbiology 65, 1578-1583.

9. Sulaiman IM et al. (2005) Unique endemicity of cryptosporidiosis in children in Kuwait. Journal of Clinical Microbiology 43, 2805-2809.

10. Hopkins RM et al. (1997) Ribosomal RNA sequencing reveals differences between the genotypes of Giardia isolates recovered from humans and dogs living in the same locality. Journal of Parasitology 83, 44-51.

11. Albert MJ et al. (1999) Case-control study of enteropathogens associated with childhood diarrhea in Dhaka, Bangladesh. Journal of Clinical Microbiology 37, 3458-3464.

12. Bilenko $\mathbf{N}$ et al. (2004) Does co-infection with Giardia lamblia modulate the clinical characteristics of enteric infections in young children? European Journal of Epidemiology 19, 877-883.

13. Haque R et al. (2009) Prospective case-control study of the association between common enteric protozoal parasites and diarrhea in Bangladesh. Clinical Infectious Diseases 48, 1191-1197.

14. Bodhidatta L et al. (2010) Case-control study of diarrheal disease etiology in a remote rural area in Western Thailand. The American Journal of Tropical Medicine and Hygiene 83, 1106-1109.

15. Veenemans J et al. (2011) Protection against diarrhea associated with Giardia intestinalis is lost with multi-nutrient supplementation: a study in Tanzanian children. PLoS Neglected Tropical Diseases 5, e1158.
16. Wilke H and Robertson LJ (2009) Preservation of Giardia cysts in stool samples for subsequent PCR analysis. Journal of Microbiological Methods 78, 292-296.

17. Maikai BV et al. (2012) Molecular characterizations of Cryptosporidium, Giardia, and Enterocytozoon in humans in Kaduna State, Nigeria. Experimental Parasitology 131, 452-456.

18. Ogunsanya TI, Rotimi VO and Adenuga A (1994) A study of the aetiological agents of childhood diarrhoea in Lagos, Nigeria. Journal of Medical Microbiology 40, 10-14.

19. Gyang VP et al. (in press) Intestinal parasitic infections: current status and associated risk factors among school aged children in an archetypal African urban slum in Nigeria. Journal of Microbiology, Immunology and Infection. doi: 10.1016/j.jmii.2016.09.005.

20. Efunshile MA et al. (2015) Molecular detection of the carriage rate of four intestinal protozoa with real-time polymerase chain reaction: possible overdiagnosis of Entamoeba histolytica in Nigeria. The American Journal of Tropical Medicine and Hygiene 93, 257-262.

21. Anejo-Okopi JA et al. (2016) Molecular characterization of Cryptosporidium in children aged 0- 5 years with diarrhea in Jos, Nigeria. The Pan African Medical Journal 25, 253.

22. Ukwah BN et al. (2017) Cryptosporidium species and subtypes in diarrheal children and HIV-infected persons in Ebonyi and Nsukka, Nigeria. Journal of Infection in Developing Countries 11, 173-179.

23. Ayinmode AB, Fagbemi BO and Xiao L (2012) Molecular characterization of Cryptosporidium in children in Oyo State, Nigeria: implications for infection sources. Parasitology Research 110, 479-481. 